\title{
ON THE FRATTINI SUBGROUP
}

\author{
BY \\ ALICE WHITTEMORE( ${ }^{(1)}$
}

\section{Introduction.}

1.1. Statement of results. The Frattini subgroup $\Phi(G)$ of a group $G$ is the intersection of the maximal subgroups of $G$; if $G$ has no maximal subgroups $\Phi(G)=G$. The Frattini subgroup is characterized as the set of nongenerators of $G$, that is those elements $g$ of $G$ with the property that for all subgroups $T$ of $G,\langle T, g\rangle=G$ $\Rightarrow T=G$. Following Gaschütz [1], $G$ will be called $\Phi$ free if $\Phi(G)=1$.

This paper represents an attempt to relate the Frattini subgroup of a group constructed from well-known groups to the Frattini subgroup of its constituent groups; the two types of constructions considered are the free product of groups with amalgamated subgroup $\left({ }^{2}\right)$, and the wreath product of groups. Concerning the former, the following main result is proved in $\$ 2$.

THEOREM 1. The free product of finitely many free groups with cyclic amalgamation is $\Phi$ free.

In particular we have

COROLlary. If

$$
G=\left\langle a_{1}, a_{2}, \ldots, a_{n}, b_{1}, b_{2}, \ldots, b_{n} ; \prod_{i=1}^{n} a_{i}^{-1} b_{i}^{-1} a_{i} b_{i}\right\rangle
$$

is the fundamental group of a two-dimensional orientable surface, then $G$ is $\Phi$ free.

The motivation for Theorem 1 is a paper due to Graham Higman and B. H. Neumann [2] where it is shown that while the free product of nontrivial groups is $\Phi$ free, this is in general not true for a free product with amalgamations-in fact the Frattini subgroup of such a group can coincide with the amalgamated subgroup. Higman and Neumann raised the questions: Can the Frattini subgroup of a free product with amalgamation be larger than the amalgamated subgroup-indeed does such a group necessarily have maximal subgroups? That the questions remain unanswered is indicative of the difficulties inherent in the structure of these groups.

The torsion subgroup $A_{T}$ of an abelian group $A$ is the subgroup consisting of all elements of finite order. Theorem 1 can be used to prove the following statement

Received by the editors July 1, 1968.

(1) This work was sponsored in part by the National Science Foundation and was greatly facilitated by the advice and suggestions of Gilbert Baumslag.

$\left({ }^{2}\right)$ For definitions and terminology, see [5]. 
about the Frattini subgroup of a generalized free product when the factors are finitely generated abelian groups.

THEOREM 2. Let $G$ be the free product of finitely many finitely generated abelian groups with amalgamated subgroup $H$. Then $\Phi(G) \leqq H_{T}$.

In $\S 3$ we turn to wreath products. In some cases there does seem to exist a tenuous relationship between the Frattini subgroup of the wreath product $A \mathrm{Wr} B$ of two groups $A$ and $B$ and that of $A$ and $B$. For example

THEOREM 3. If $A$ and $B$ are finite groups of coprime order then $A$ wr $B$ is $\Phi$ free $\Leftrightarrow A$ is $\Phi$ free.

And on the other hand

THEOREM 4. If $A$ and $B$ are groups without maximal subgroups and $A$ is abelian, then both $A$ Wr $B$ and $A$ wr $B$ have no maximal subgroups.

In particular, if $Q$ is the additive group of rationals, then $Q \mathrm{Wr} Q$ has no maximal subgroups.

1.2. Preliminaries. If $H$ is a subgroup of $G$ we write $H \leqq G$, and $H \unlhd G$ when $H$ is normal in $G ; H<G(H \triangleleft G)$ denotes proper inclusion. The set of elements of $G$ which do not belong to $H$ is written $G \backslash H$. The normal closure of $H$ in $G$ is the least normal subgroup of $G$ containing $H$ and will be denoted by $n m_{G} H$. A subgroup $K$ of $H$ will be called $G$ normal if $K$ is normal in $G$. A group is residually $\Phi$ free if its normal subgroups with $\Phi$ free quotient group intersect in the identity.

It is easily verified that $\Phi(G)$ is characteristic in $G$; moreover if $N$ is a normal subgroup of $G$ then $\Phi(G) N / N \leqq \Phi(G / N)$ and hence $\Phi(G / N)=1 \Rightarrow \Phi(G) \leqq N$. From this it follows that any residually $\Phi$ free group is itself $\Phi$ free and hence free abelian groups, being residually prime cycles, are $\Phi$ free.

Stronger than the notion of nongenerators is that of omissibility. A subset $S$ of a group $G$ is called omissible in $G$ if for each subgroup $T$ of $G,\langle T, S\rangle=G \Rightarrow T=G$. An omissible subset is always contained in $\Phi(G)$, but unless $\Phi(G)$ is finitely generated, it need not be omissible.

\section{The proof of Theorems 1 and 2.}

2.1. We begin with a proposition that provides a partial answer to the second of Higman and Neumann's questions.

Proposition 2.1. Let $\Lambda$ be an indexing set of cardinality greater than one, let $G=\prod_{\lambda \in \Lambda}^{*}\left(G_{\lambda} ; H_{\lambda}\right)$ with the amalgamated subgroup denoted by $H$ and suppose that the normal closure of $H_{\lambda}$ in $G_{\lambda}$ is properly contained in $G_{\lambda}$ for at least two $\lambda \in \Lambda$. Then $\Phi(G)$ is contained in the normal closure of $H$ in $G$.

Proof. If we denote the free product $\prod_{\lambda \in \Lambda}^{*} G_{\lambda} / n m_{G_{\lambda}} H_{\lambda}$ by $G^{*}$, then by Higman and Neumann's result on the $\Phi$ freeness of a free product and the remarks of $\S 1.2$ it will suffice to show that $G / n m_{G} H \cong G^{*}$. The homomorphisms $\theta_{\lambda}$ mapping $G_{\lambda}$ 
into $G^{*}$ defined by $\theta_{\lambda}: G_{\lambda} \rightarrow G_{\lambda} / n m_{G_{\lambda}} H_{\lambda}$ agree on the elements of $H$ (that is $h \theta_{\lambda}=h \theta \mu$ for $\lambda, \mu \in \Lambda, h \in H$ ); under these conditions [5] the $\theta_{\lambda}$ can be extended to a homomorphism $\theta$ mapping $G$ onto $G^{*}$. We check that ker $\theta=n m_{G} H$. If $g \in n m_{G} H$ then $g=\prod_{i=1}^{n} h_{i}^{g_{i}}\left(g_{i} \in G, h_{i} \in H, 1 \leqq i \leqq n\right)$, hence $g \theta=1$. On the other hand if an element $g$ in the kernel of $\theta$ is put in normal form $g=g_{1}, g_{2} \cdots g_{n} h$ where $g_{i} \in G_{\lambda_{i}} \mid H$, $h \in H, \lambda_{i} \neq \lambda_{i+1}$, we have $1=\left(g_{1} g_{2} \cdots g_{n} h\right) \theta=g_{1} \theta g_{2} \theta \cdots g_{n} \theta$ where $g_{i} \theta \in G_{\lambda_{i}} / n m_{G_{\lambda_{i}}} H_{\lambda_{i}}$ and $\lambda_{i} \neq \lambda_{i+1}$ which implies, since $G^{*}$ is a free product, that $g_{i} \theta=1$ for $1 \leqq i \leqq n$, i.e. that $g_{i} \in n m_{G_{\lambda_{i}}} H$. Hence $g \in n m_{G} H$.

2.2. The next proposition is a special case of the theorem, when each of the free factors is cyclic. In both Propositions 2.2 and 2.3 the characterization of $\Phi(G)$ as the set of nongenerators of $G$ is used to show that $G$ is $\Phi$ free. We produce for any given element we wish to exclude from $\Phi(G)$ a proper subgroup $T$ of $G$ which, together with the element in question, generates all of $G$.

Proposition 2.2. The free product with amalgamations of finitely many infinite cyclic groups is $\Phi$ free.

Proof. Let $X_{1}, X_{2}, \ldots, X_{n}$ be infinite cyclic groups with $X_{i}=\left\langle x_{i}\right\rangle, H_{i}=\left\langle x_{i}^{m_{i}}\right\rangle$ ( $m_{i}$ integers greater than one), $1 \leqq i \leqq n$, and let $G=\prod_{i=1}^{* n}\left(X_{i} ; H_{i}\right)$ with amalgamated subgroup $H$. By Proposition $2.1 \Phi(G) \leqq H$; hence it suffices to exclude an arbitrary element $h=x_{i}^{\lambda m_{i}}(1 \leqq i \leqq n, \lambda>0)$ of $H$ from $\Phi(G)$. For this purpose choose a prime $p>1$ such that $\left(p, \lambda m_{i}\right)=1$ for all $i$ and let $T=\left\langle x_{1}^{p}, x_{2}^{p}, \ldots, x_{n}^{p}\right\rangle$. Clearly $\langle T, h\rangle=G$; it remains to show that $T \neq G$. To see this let $Y_{i}=\left\langle x_{i}^{p}\right\rangle$ and note that for $1 \leqq j, k \leqq n$, $Y_{j} \cap H=Y_{k} \cap H$, hence [5] the subgroups $Y_{1}, Y_{2}, \ldots, Y_{n}$ generate their free product amalgamating their common intersection with $H$, and $T=\prod_{i=1}^{* n}\left(Y_{i}\right.$; $\left.Y_{i} \cap H_{i}\right)$. If $x_{1}$ were in $T$, since $x_{1} \notin Y_{1}$ the normal form for $x_{1}$ in $T$ must have length $\geqq 2$, say $x_{1}=x_{i_{1}}^{r_{1} p} x_{i_{2}}^{r_{2} p} \cdots x_{i_{l}}^{r_{l} p}$ where $x_{i_{k}}^{r_{k} p} \in Y_{i_{k}} \mid H_{i_{k}}(1 \leqq k \leqq l, l \geqq 2)$ and $i_{k} \neq i_{k+1}$ $(1 \leqq k<l)$, i.e. $1=x_{1}^{-1} x_{i_{1}}^{r_{1} p} \cdots x_{i_{l}}^{r_{l} p}$. If $x_{i}^{-1} x_{i_{1}^{1}}^{r_{1} p} \notin H$ we have in $G$ a product of terms lying outside of $H$ such that no two successive terms belong to the same factor equal to one, which is impossible [5]. If $i_{1}=1$ and $x_{i}^{r_{1}}{ }^{p-1} \in H$ then $x_{1}^{r_{1} p-1} x_{i_{2}^{2}}^{r_{2} p} \in Y_{i_{2}} \backslash H$ giving the same contradiction. Thus $x_{1} \notin T$ and $h \notin \Phi(G)$.

2.3. When certain restrictions are placed on an arbitrary free product with amalgamations the above argument can again be used to show that the group is $\Phi$ free.

Proposition 2.3. If in a free product $G$ with amalgamated subgroup $H$ there exists an element which conjugates each nontrivial element of $H$ outside of $H$, then $G$ is $\Phi$ free.

Proof. Let $G=\prod_{\lambda \in \Lambda}^{*}\left(G_{\lambda} ; H_{\lambda}\right)$ and let $\mu$ be an element of the nonempty indexing set $\Lambda$. If $A=G_{\mu}, H_{A}=H_{\mu}$ and $B=\prod_{\lambda \in \Lambda ; \lambda \neq \mu}^{*}\left(G_{\lambda} ; H_{\lambda}\right)$ with $H_{B}$ the amalgamated subgroup of $B$, then $G=\left(A * B ; H_{A}=H_{B}\right)$; hence it is enough to prove the proposition for the free product with amalgamations of two groups $A$ and $B$. So let $G=(A * B ; H)$ and let $c \in G$ be such that $h^{c} \notin H$ for all nontrivial $h$ in $H$. Note 
then that $h^{c-1} \notin H$ for all nontrivial $h$ in $H$. We use the normality of $\Phi(G)$ to conclude that since no nontrivial subgroup of $H$ is normal in $G$, it is enough to exclude elements of length $\geqq 1$ from $\Phi(G)$, and secondly, in order to exclude an arbitrary element $g$ in $G$ of length at least one, it is enough to exclude any conjugate of $g$ by an element of $G$ (or, in fact, any power of $g$ or $g^{-1}$ ). We consider four cases, corresponding to the four possibilities for a normal form for $c$.

Case I. $c=\alpha_{1} \beta_{1} \cdots \alpha_{m} \beta_{m}$ where $\alpha_{i}, \beta_{i} \in A \backslash H, B \backslash H$ respectively $(1 \leqq i \leqq m)$ and $m \geqq 1$. If $g$ is an arbitrary element of $G$ of length at least one, conjugation of $g$ by a suitable element of $G$ will yield one of the two normal forms (i) $\bar{\beta}_{1} \bar{\alpha}_{1} \cdots \bar{\beta}_{n} \bar{\alpha}_{n} \bar{\beta}_{n+1}$ or (ii) $\bar{a}_{1} \bar{\beta}_{1} \cdots \bar{a}_{n} \bar{\beta}_{n}$, where $n \geqq 1$; hence we may assume that $g$ is in one of these two forms $\left(\bar{a}_{i}, \bar{\beta}_{i} \in A \backslash H, B \backslash H\right.$ respectively, $\left.1 \leqq i \leqq n\right)$.

(i) If $g=\bar{\beta}_{1} \bar{a}_{1} \cdots \bar{\beta}_{n} \bar{a}_{n} \bar{\beta}_{n+1}$ we exclude $z=g^{c \alpha_{1} c^{-1}}$ from $\Phi(G)$. If $T=\left\langle z^{-1} A z, B\right\rangle$ it is clear that $\langle T, z\rangle=G$. If $z \in T, z$ would equal a product of terms coming alternately out of $z^{-1} A z$ and $B$ and multiplication of both sides by $z^{-1}$ would yield one of the following equations, where we may assume the $a_{i}$ and $b_{j}$ are nontrivial:

(a) $1=a_{1}^{z} b_{1} a_{2}^{z} b_{2} \cdots a_{k}^{z} b_{k} z^{-1}(k \geqq 1)$,

(b) $1=b_{1}\left(a_{1}^{z} b_{2} \cdots a_{k}^{z} b_{k+1} z^{-1}\right)(k \geqq 0)$,

(c) $1=\left(a_{1}^{z} b_{1} a_{2}^{z} b_{2} \cdots a_{k}^{z} b_{k} z^{-1}\right) a_{k+1}(k \geqq 0)$,

(d) $1=b_{1}\left(a_{1}^{z} b_{2} \cdots a_{k}^{z} b_{k+1} z^{-1}\right) a_{k+1}(k \geqq 0)$.

Equation (a) becomes

$$
\begin{aligned}
1= & c \alpha_{1}^{-1} c^{-1} g^{-1}\left(c \alpha_{1} c^{-1} a_{1} c \alpha_{1}^{-1} c^{-1}\right) g c \alpha_{1}\left(c^{-1} b_{1} c\right) \alpha_{1}^{-1} c^{-1} g^{-1}\left(c \alpha_{1} c^{-1} a_{2} c \alpha_{1}^{-1} c^{-1}\right) \\
& \cdot g c \alpha_{1} c^{-1} b_{2} \cdots c \alpha_{1}^{-1} c^{-1} g^{-1}\left(c \alpha_{1} c^{-1} a_{k} c \alpha_{1}^{-1} c^{-1}\right) g c \alpha_{1}\left(c^{-1} b_{k} c\right) \alpha_{1}^{-1} c^{-1} g^{-1} c \alpha_{1} c^{-1} .
\end{aligned}
$$

But if $a_{1} \in H$ then $a_{1}^{c}$ is a word beginning and ending with a term from $B \backslash H$ which

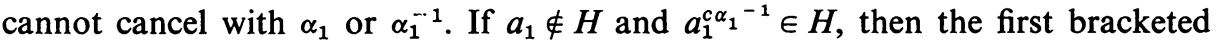
expression begins and ends with a term from $A \backslash H$ which will not cancel with $\bar{\beta}_{1}$ or $\bar{\beta}_{1}^{-1}$. Similarly, if $b_{1} \in H$ then $b_{1}^{c}$ begins and ends with a term from $B \backslash H$, while if $b_{1} \in B \backslash H$ no cancellation can occur at all. Continuing this process of combining terms we see that at each $a_{i}$ and $b_{j}$ after combining at most seven terms we are left with an expression with factors coming alternately out of $A \backslash H$ and $B \backslash H$, and that the terms consumed at an $a_{i}$ or $b_{j}$ never overlap. Hence the expression in (1) cannot collapse to 1 . Moreover, with closer scrutiny we observe that even if the right-hand side of equation (1) were preceded with a nontrivial element $b \in B$ and/or followed by a nontrivial element $a \in A$, the cancellation stops at $c$ and $c^{-1}$; hence equations (b) through (d) cannot occur. From this it follows that $z \notin T$, i.e. that $z$ and hence $g \notin \Phi(G)$.

(ii) If $g=\bar{a}_{1} \bar{\beta}_{1} \cdots \bar{a}_{n} \bar{\beta}_{n}$ choose an integer $\varepsilon$ such that $\varepsilon n>m$. Then $l\left(g^{\varepsilon}\right)=2 \varepsilon n$ $>2 m=l(c)$, and

$$
c g^{\varepsilon} c^{-1}=\alpha_{1} \beta_{1} \cdots \alpha_{m} \beta_{m} \bar{a}_{1} \bar{\beta}_{1} \cdots \bar{a}_{n} \bar{\beta}_{n} \cdots \bar{\alpha}_{1} \bar{\beta}_{1} \cdots \bar{a}_{n} \bar{\beta}_{n} \beta_{m}^{-1} \alpha_{m}^{-1} \cdots \beta_{1}^{-1} \alpha_{1}^{-1} .
$$

Cancel and combine as much as possible at the only spot where cancellation can occur-between $\bar{\beta}_{n}$ and $\beta_{m}^{-1}$. Note that $c$ remains untouched. In fact at worst one 
is left with $c$ followed by two terms coming out of $A \backslash H$ and $B \backslash H$, say $c g^{\varepsilon} c^{-1}=c \alpha \beta$. If after cancellation and contraction $c g^{\varepsilon} c^{-1}$ ends in a $\beta \in B \backslash H$, i.e. $c g^{\varepsilon} c^{-1}=c \alpha \cdots \beta$, square it to obtain $c \alpha \cdots \beta c \alpha \cdots \beta=z$. We exclude $z$ from $\Phi(G)$; for this purpose let $T=\left\langle z^{-1} B z, A\right\rangle$. Again under the assumption that $z \in T$ we obtain one of the following equations:

(a') $1=b_{1}^{z} a_{1} b_{2}^{z} a_{2} \cdots b_{k}^{z} a_{k} z^{-1}(k \geqq 1)$,

(b') $1=a_{1}\left(b_{1}^{z} a_{2} \cdots b_{k}^{z} a_{k+1} z^{-1}\right)(k \geqq 0)$,

(c') $1=\left(b_{1}^{z} a_{1} b_{2}^{z} a_{2} \cdots b_{k}^{z} a_{k} z^{-1}\right) b_{k+1}(k \geqq 0)$,

(d') $1=a_{1}\left(b_{1}^{z} a_{2} \cdots b_{k}^{z} a_{k+1} z^{-1}\right) b_{k+1}(k \geqq 0)$.

As in the preceding case we examine only equation $\left(a^{\prime}\right)$; that equations $\left(b^{\prime}\right)$ through $\left(d^{\prime}\right)$ cannot occur will follow as before from the limited contractions possible in this equation.

Equation $\left(a^{\prime}\right)$ becomes

$$
\begin{gathered}
1=\beta^{-1} \cdots \alpha^{-1} c^{-1} \beta^{-1} \cdots \alpha^{-1}\left(c^{-1} b_{1} c\right) \alpha \cdots \beta\left(c \alpha \cdots \beta a_{1} \beta^{-1} \cdots \alpha^{-1} c^{-1}\right) \beta^{-1} \\
\cdots \alpha^{-1}\left(c^{-1} b_{2} c\right) \alpha \cdots \beta c \alpha \cdots \beta a_{2} \cdots \beta^{-1} \cdots \alpha^{-1} c^{-1} \beta^{-1} \\
\cdots \alpha^{-1}\left(c^{-1} b_{k} c\right) \alpha \cdots \beta\left(c \alpha \cdots \beta a_{k} \beta^{-1} \cdots \alpha^{-1} c^{-1}\right) \beta^{-1} \cdots \alpha^{-1} c^{-1} .
\end{gathered}
$$

As the bracketed expressions involving $b_{i}$ begin and end with terms coming from $B \backslash H$ and those involving $a_{i}$ begin and end with terms coming from $A \backslash H$, the entire expression in $\left(2^{\prime}\right)$ cannot collapse to 1 , implying as before that $g \notin \Phi(G)$.

If after cancellation in (2) $c g^{\varepsilon} c^{-1}=c \cdots \alpha$ where $\alpha \in A \backslash H$, let $z=c^{-1} \beta_{1} c g^{\varepsilon} c^{-1} \beta_{1}^{-1} c$ $=c^{-1} \beta_{1} c \cdots \alpha \beta_{1}^{-1} c$, and let $T=\left\langle z^{-1} A z, B\right\rangle . z \in T$ only if an equality of type (a), (b), (c) or (d) holds; restricting our attention as before to equation (a) we have

$$
\begin{aligned}
1= & c^{-1} \beta_{1} \alpha^{-1} \cdots c^{-1} \beta_{1}^{-1}\left(c a_{1} c^{-1}\right) \beta_{1}\left(c \cdots \alpha \beta_{1}^{-1} c b_{1} c^{-1} \beta_{1} \alpha^{-1} \cdots c^{-1}\right) \\
& \cdot \beta_{1}^{-1}\left(c a_{2} c^{-1}\right) \beta_{1} c \cdots \alpha \beta_{1}^{-1} c b_{2} \cdots c^{-1} \beta_{1} \alpha^{-1} \cdots c^{-1} \beta_{1}^{-1}\left(c a_{k} c^{-1}\right) \\
& \cdot \beta_{1}\left(c \cdots \alpha \beta_{1}^{-1} c b_{k} c^{-1} \beta_{1} \alpha^{-1} \cdots c^{-1}\right) \beta_{1}^{-1} c .
\end{aligned}
$$

Consider the bracketed expressions involving the $a_{i}$. If $a_{i} \notin H$ no cancellation can occur at all; if $a_{i} \in H$ then $a_{i}^{c-1}$ begins and ends with a term from $A \backslash H$, hence cannot cancel with $\beta_{1}$ or $\beta_{1}^{-1}$. Similarly, in those bracketed expressions involving $b_{i}$, if $b_{i} \in H$ cancellation stops at $b_{i}^{c^{-1}}$. If $b_{i} \notin H$ and $b_{i}^{c} \in H$, cancel and combine as much as possible, noting that at worst the bracketed expressions will be of the form chc ${ }^{-1}$ (where $h \in H$ ), which begins and ends with terms from $A \backslash H$; hence cannot combine with $\beta_{1}$ or $\beta_{1}^{-1}$. We again conclude that $z \notin T$ and $g \notin \Phi(G)$. Thus if $c$ is in form I, $\Phi(G)=1$.

Case II. $c=\beta_{1} \alpha_{1} \cdots \beta_{m} \alpha_{m}\left(\alpha_{i}, \beta_{i} \in A \backslash H, B \backslash H\right.$ respectively for $\left.1 \leqq i \leqq m ; m \geqq 1\right)$. Since by conjugating $g$ (where $g$ is an arbitrary element of $G$ subject to $l(g) \geqq 1$ ) by a suitable element of $G$ we may also assume that either (i') $g=\bar{a}_{1} \bar{\beta}_{1} \cdots \bar{\alpha}_{n} \bar{\beta}_{n} \bar{\alpha}_{n+1}$ $(n \geqq 0)$ or (ii') $g=\bar{\beta}_{1} \bar{\alpha}_{1} \cdots \bar{\beta}_{n} \bar{\alpha}_{n}(n \geqq 1)$. Case II follows from Case I by interchanging $A$ and $B$. 
Case III. $c=\alpha_{1} \beta_{1} \cdots \alpha_{m} \beta_{m} \alpha_{m+1} \quad\left(\alpha_{i}, \beta_{j} \in A \backslash H, B \backslash H\right.$ respectively, $1 \leqq i \leqq m+1$, $1 \leqq j \leqq m, m \geqq 0$ ).

Again, given an element $g$ in $G$ of length at least one we conjugate it judiciously to obtain (i) or (ii). If $g$ can be put in form (i), that is, $\bar{\beta}_{1} \bar{\alpha}_{1} \cdots \bar{\beta}_{n} \bar{\alpha}_{n} \bar{\beta}_{n+1}$, we exclude $z=g^{c^{-1} \beta_{1} c}$ by choosing $T=\left\langle z^{-1} A z, B\right\rangle$ and observing that while $\langle T, z\rangle=G$, if $z \in T$ one of the equations (a) through (d) must hold, or, again confining ourselves to (a) and expanding,

$$
\begin{aligned}
1= & c^{-1} \beta_{1}^{-1} c g^{-1}\left(c^{-1} \beta_{1} c a_{1} c^{-1} \beta_{1}^{-1} c\right) g c^{-1} \beta_{1}\left(c b_{1} c^{-1}\right) \\
& \cdot \beta_{1}^{-1} c g^{-1}\left(c^{-1} \beta_{1} c a_{2} c^{-1} \beta_{1}^{-1} c\right) g c^{-1} \beta_{1} c b_{2} \\
& \cdots c^{-1} \beta_{1}^{-1} c g^{-1}\left(c^{-1} \beta_{1} c a_{k} c^{-1} \beta_{1}^{-1} c\right) g c^{-1} \beta_{1}\left(c b_{k} c^{-1}\right) \beta_{1}^{-1} c g^{-1} \beta_{1} c .
\end{aligned}
$$

Note that after all possible cancellations are performed the bracketed expressions begin and end with terms from $A \backslash H$ and we are left with a nontrivial expression. We complete the argument as before.

To eliminate $g$ qua form (ii), i.e. $\bar{\alpha}_{1} \bar{\beta}_{1} \cdots \bar{\alpha}_{n} \bar{\beta}_{n}$, from $\Phi(G)$ we again choose $k$ such that $\varepsilon n>m$, let

$$
\begin{aligned}
z=c g^{-1} c^{-1}= & \alpha_{1} \beta_{1} \cdots \alpha_{m} \beta_{m} \alpha_{m+1}\left(\bar{\beta}_{n}^{-1} \cdots \bar{\alpha}_{1}^{-1} \cdots \bar{\beta}_{n}^{-1} \cdots \bar{\alpha}_{1}^{-1}\right) \\
& \cdot \alpha_{m+1}^{-} \beta_{m}^{-1} \alpha_{m}^{-1} \cdots \beta_{1}^{-1} \alpha_{1}^{-1}
\end{aligned}
$$

and apply the argument used in Case I (ii), since the only essential difference between (5) and (2) is that in (5) at worst, $2 m+1$ terms of $g^{-\varepsilon}$ are consumed after cancellation; $c$ however still remains intact.

Case IV. $c=\beta_{1} \alpha_{1} \cdots \beta_{m} \alpha_{m} \beta_{m+1}(m \geqq 0)$. In this case we conjugate our arbitrary $g$ of nonzero length into forms (i') or (ii') by a suitable element of $G$ and, interchanging $A$ and $B$, apply Case III.

Since we have exhausted all the possibilities for $c$, the proposition follows.

2.4. We are now in a position to deduce Theorem 1 . We use the following simple lemmas:

LEMMA 2.4.1. Let $g$ and $h$ be elements in a free group $F$. If $g^{-1} h^{m} g=h^{n}$ then $m=n$ and $g$ and $h$ commute.

Proof. The group $K=\langle g, h\rangle$ is free of rank either one or two. If $K$ is free of rank one, no proof is required. But in a free group of rank two any two generators are free generators [3], hence $g$ and $h$ freely generate $K$ and the lemma follows.

We use this in the proof of

LEMMA 2.4.2. Let $F$ be a free group on a set of free generators $X=\left\{x_{1}, x_{2}, \ldots\right\}$ where $X$ may have any cardinality greater than one, and let $H=\langle h\rangle$ be a cyclic subgroup of $F$. Then there exists $c \in F$ such that $\left(h^{n}\right)^{c} \notin H$ for all nonzero integers $n$.

Proof. By Lemma 2.4.1 it is enough to produce an element $c$ such that $[h, c] \neq 1$. Express $h$ uniquely as a word $w\left(x_{i}\right)$ in the given generators for $F$ and let $S$ be the subset of $X$ consisting of those $x_{i}$ that appear in $w\left(x_{i}\right)$. If there exists an $x_{k} \in X \mid S$ 
then $\left[h, x_{k}\right] \neq 1$. If $S=X$ then $w\left(x_{i}\right)$ involves at least two free generators, either one of which will not commute with $h$.

Collecting our results we now have that the free product of finitely many free groups with cyclic amalgamation is $\Phi$ free. For by Proposition 2.2 we may assume that one of the free factors is noncyclic, which implies by Lemma 2.4.2 that the hypothesis of Proposition 2.3 is satisfied.

2.5. That the theorem is false if we allow $G$ to be the generalized free product of infinitely many free groups is illustrated with the following example due to Gilbert Baumslag. Consider the group $G=\left\langle a_{1}, a_{2}, a_{3}, \ldots, a_{i}, \ldots ; a_{1}^{2}=a_{2}^{3}=a_{3}^{5}=\cdots=a_{i}^{p_{t}}\right.$ $=\cdots\rangle$ where $p_{i}$ denotes the $i$ th positive prime. $G$ is the free product of countably many infinite cyclic groups with amalgamated subgroup $H$; let $H=\langle h\rangle$. We know $\Phi(G) \leqq H$; to show $\Phi(G)$ actually coincides with $H$ we show $h \in \Phi(G)$. Suppose not and let $M$ be a maximal subgroup of $G$ excluding $h$. Then, since $h$ is central in $G$, we have for elements $m_{i} \in M, a_{i}=m_{i} h_{i}^{\alpha_{i}}$ and $m_{i}^{p_{i}}=h^{1-p_{i} \alpha_{i}}$. Now let $p_{i_{1}}, p_{i_{2}}, \ldots, p_{i_{n}}$ be the prime divisors of $1-2 \alpha_{1}$. Then $1-2 \alpha_{1}, 1-p_{i_{1}} \alpha_{i_{1}}, \ldots, 1-p_{i_{n}} \alpha_{i_{n}}$ are relatively prime, whence there exist $\beta, \beta_{1}, \ldots, \beta_{n}$ such that $\left(1-2 \alpha_{1}\right) \beta+\left(1-p_{i_{1}} \alpha_{i_{1}}\right) \beta_{1}+\cdots$ $+\left(1-p_{i_{n}} \alpha_{i_{n}}\right) \beta_{n}=1$. But then

$$
m_{i}^{2 \beta} m_{i_{1} 1}^{p_{i} \beta_{1}} \cdots m_{i_{n} n}^{p_{1} \beta_{n}}=h^{\left(1-2 \alpha_{1}\right) \beta+\left(1-p_{i_{1}} \alpha_{i_{1}}\right) \beta_{1}+\ldots+\left(1-p_{i_{n}} \alpha_{i_{n}}\right) \beta_{n}}=h
$$

which puts $h$ in $M$, a contradiction which establishes that $\Phi(G)=H$.

\subsection{Proof of Theorem 2 .}

LEMMA 2.6.1 Let $G$ be the free product of finitely generated abelian groups $A_{1}, A_{2}, \ldots, A_{n}(n<\infty)$ with amalgamated subgroup $H$ and let $h$ be an element of $H$ with infinite order. Then there exists a homomorphism $\phi$ mapping $G$ onto the free product $P$ of $n$ finitely generated free abelian groups with cyclic amalgamation such that $h \notin \operatorname{ker} \phi$.

Proof. If $a_{1}, a_{2}, \ldots, a_{s}$ is a basis for $H$ we have, since $h$ has infinite order, that $h=a_{1}^{\varepsilon_{1}} a_{2}^{\varepsilon_{2}} \cdots a_{s}^{\varepsilon_{s}}$ where say, $\varepsilon_{1} \neq 0$ and $a_{1}$ has infinite order. For $1 \leqq i \leqq n$ let $N_{i}$ $=\left\langle A_{i_{T}}, a_{2}, \ldots, a_{s}\right\rangle$ and let $\phi_{i}$ map each of the subgroups $A_{i}$ of $G$ onto the free abelian groups $A_{i} / N_{i}$. If $P$ is the free product of the groups $A_{i} / N_{i}$ amalgamating the subgroups generated by the elements $a_{1} N_{i}$, then it is easily checked that the $\phi_{i}$, regarded as maps into $P$, agree on $H$ and may thus be extended to a map $\phi$ of $G$ onto $P$ with the required property that $h \phi \neq 1$.

LEMMA 2.6.2. The free product $P$ of finitely generated free abelian groups $A_{1}, A_{2}, \ldots, A_{n}(n<\infty)$ with cyclic amalgamation $C$ is $\Phi$ free.

Proof. One can choose basis $a_{i 1}, a_{i 2}, \ldots, a_{i r(i)}$ for the groups $A_{i}$ such that the cyclic subgroup $C=\left\langle a_{i 1}^{\varepsilon_{1}}\right\rangle$. Let $N_{i}=\left\langle a_{i 2}, \ldots, a_{i r(i)}\right\rangle$, let $\sigma_{i}: A_{i} \rightarrow A_{i} / N_{i}$ and let $\hat{P}$ be the free product of the infinite cycles $A_{i} / N_{i}$ amalgamating the subgroups generated by the elements $a_{i 1}^{\varepsilon_{i}} N_{i}$. As in Lemma 2.6.1 we note that since the $\sigma_{i}$, regarded as maps into $\hat{P}$, agree on $C$ we may extend them to a map $\sigma$ of $P$ onto $\hat{P}$, 
such that $C \cap \operatorname{ker} \sigma=1$. But by Proposition $2.2 \hat{P}$ is $\Phi$ free and hence $\Phi(P) \leqq \operatorname{ker} \sigma$. Since by Proposition $2.1 \Phi(P) \subseteq C$, the lemma follows.

Theorem 2 now follows immediately. For if $G$ is the free product of finitely many finitely generated abelian groups with amalgamated subgroup $H$, by Proposition 2.1 $\Phi(G) \leqq H$. But if $h$ is an arbitrary element of $H$ of infinite order, by 2.6.1 there exists a homomorphism $\phi$ mapping $G$ onto a group $P$ such that $h \notin \operatorname{ker} \phi$. Since by $2.6 .2 P$ is $\Phi$ free we have $h \notin \operatorname{ker} \phi \supseteq \Phi(G)$ as required.

Note that in 2.6.1 and 2.6.2 the amalgamated subgroups of $P$ and $\hat{P}$ may coincide with one of the factors allowing the possibility that $P$ and $\hat{P}$ are themselves free abelian. But since free abelian groups are $\Phi$ free the desired result follows.

That Theorem 2 is the best possible result with respect to the $\Phi$ freeness of such a group is illustrated by the following remark due to John Campbell:

2.6.3. If a group $G$ contains a normal subgroup $N$ and a subgroup $K$ with the property that $N$ is omissible in $K$, then $N$ is omissible in $G$.

For if not, then $G=N T$ with $T<G$. Since $N \$ T$ and since by assumption $N \subseteq K$ we have $N \$ T \cap K<K$. But then $K=N(T \cap K)$ which contradicts the omissibility of $N$ in $K$.

In particular when $G$ is the free product of a family of groups $\left\{G_{\lambda}\right\}_{\lambda \in \Lambda}$ with amalgamated subgroup $H$ and $N$ is a finitely generated subgroup of $H$ such that $N \triangleleft G$ and $N \leqq \Phi\left(G_{\lambda}\right)$ for some $\lambda \in \Lambda$ then $N \leqq \Phi(G)$. And in the case when the groups $G_{\lambda}$ are finitely generated and abelian, if $H$ intersects the Frattini subgroup of any one of the constituent groups nontrivially, this intersection, a torsion group, is contained in $\Phi(G)$.

If in 2.6.3 we place different demands on the normal subgroup $N$ we have another source of omissible subgroups.

2.6.4. If a group $G$ contains a normal nilpotent subgroup $N$ and a subgroup $K$ such that $N \leqq \Phi(K)$ and $N$ is the normal closure in $K$ of finitely many elements, then $N$ is omissible in $G$.

Proof. By 2.6.3 it is enough to show that $N$ is omissible in $K$. To see this, let $N=n m_{K}\left(x_{1}, \ldots, x_{n}\right)$ and suppose $K=T N$ for some subgroup $T$ of $K$. Then for $x_{i}(1 \leqq i \leqq n)$ and for each $k \in K$ we have $x_{i}^{k}=x_{i}\left[x_{i}, k\right]=x_{i}\left[x_{i}, t n\right]=x_{i}\left[x_{i}, t\right]\left[x_{i}^{t}, n\right]$ $(t \in T, n \in N)$ where $\left[x_{i}^{t}, n\right] \in N^{\prime}$ which is omissible as a subgroup of the nilpotent group $N$ [4]; hence $N=\left\langle x_{1}, x_{2}, \ldots, x_{n},\left[x_{i}, t\right]\right\rangle$ where $t \in T, i=1, \ldots, n$ and $K=\left\langle x_{1}, \ldots, x_{n}, T\right\rangle=T$ as was required.

\section{Frattini subgroups of wreath products.}

3.1. Definitions. If $A$ and $B$ are groups let $K=A^{(B)}$ be the group of all functions from $B$ to $A$ with multiplication defined componentwise: if $f, g \in K, f g(b)=f(b) g(b)$ for all $b \in B$. The wreath product $A \mathrm{Wr} B$ of $A$ by $B$ is defined as a splitting extension of $K$ by $B$ using the following action: for $f \in K$ and $b \in B$ we define $f^{b} \in K$ by $f^{b}\left(b^{\prime}\right)=f\left(b^{\prime} b^{-1}\right)$ for all $b^{\prime} \in B$. We denote $A$ Wr $B$ by $W$ and refer to $K$ as the base group of $W$. If $K_{1}=\{f \in K \mid f(b) \neq 1$ for finitely many $b \in B\}$ then $K_{1} B$ forms a subgroup 
of $A$ Wr $B$ called the restricted wreath product of $A$ and $B$ and denoted by $A$ wr $B$. The Fitting subgroup $\Psi(G)$ of a group $G$ is the product of the normal nilpotent subgroups of $G$. Fitting has shown that $\Psi(G)$ is itself normal in $G$ and when $G$ is finite $\psi(G)$ is nilpotent. We will use the fact that the Fitting subgroup of a direct product of groups is the direct product of the Fitting subgroups; that is if $G=\prod_{\lambda \in \Lambda} G_{\lambda}$ then $\Psi(G)=\prod_{\lambda \in \Lambda} \Psi\left(G_{\lambda}\right)$. That $\prod_{\lambda \in \Lambda} \Psi\left(G_{\lambda}\right) \leqq \Psi(G)$ is trivial; on the other hand if $F$ is a normal nilpotent subgroup of $G$ then for each $\lambda \in \Lambda$ the projection $\pi_{\lambda}: F \rightarrow G_{\lambda}$ defined by $f \pi_{\lambda}=f(\lambda)$ maps $F$ onto a nilpotent normal subgroup of $G_{\lambda}$ which is thus contained in $\Psi\left(G_{\lambda}\right)$. Therefore $F$ and hence $\Psi(G)$ is contained in $\prod_{\lambda \in \Lambda} \Psi\left(G_{\lambda}\right)$.

The socle $S(G)$ of a finite group $G$ is the product of the minimal normal subgroups $G_{\mu}$ of $G$. It is easily verified that $S(G)$ is the direct product of suitable $G_{\mu}$ and that any $G$ normal subgroup of $S(G)$ has a $G$ normal complement in $S(G)$. Let $S(G)_{\alpha}$ be the maximal soluble $G$ normal subgroup of $S(G)$. Then for each minimal normal subgroup $G_{\mu}$ of $G$ either $G_{\mu} \cap S(G)_{\alpha}=1$ or $G_{\mu} \leqq S(G)_{\alpha}$; hence $S(G)_{\alpha}$ is the direct product of the minimal normal subgroups $G_{\mu}$ contained in it and the latter as subgroups of a soluble group are soluble. Moreover they are all direct products of prime cycles for if not they would contain characteristic subgroups, contradicting their minimality. Hence $S(G)_{\alpha}$ is the direct product of prime cycles and is called the abelian component of the socle of $G$. We note two results of Gaschütz [1] for finite groups:

3.1.1. $\Phi(G)=1 \Rightarrow \Psi(G)=S(G)_{\alpha}$, and

3.1.2. $\Phi(G)=1$ if and only if $G$ splits over $S(G)_{\alpha}$.

3.2. Proof of Theorem 3. To show that when $A$ and $B$ have coprime order $\Phi(W)=1$ if and only if $\Phi(A)=1$ we use two lemmas, the first of which is due to John Cossey.

Lemma 3.2.1. Let $W=A$ wr $B$ with $A$ and $B$ finite groups of coprime order and let $H$ be a normal subgroup of $W$. Then $H \cap K$ is complemented in $H$ by a subgroup $C$ of $B$.

Proof. Since $H \cap K$ has order prime to its index in $H$, by the Schur-Zassenhaus results $H$ splits over $H \cap K$. If $\bar{C}$ is a complement for $H \cap K$ in $H$, we have $K \bar{C}=K H$. If $C=B \cap K H$ then $K C \leqq K H$, but on the other hand if $k h \in K H$ then since $k h=k_{1} b\left(k_{1} \in K, b \in B\right)$ we have $b=k_{1}^{-1} k h \in C$ and $K H \leqq K C$. Hence $K \bar{C}=K H$ $=K C$. Since $K$ has order prime to its index in $K C$ the two complements $\bar{C}$ and $C$ for $K$ in $K C$ are conjugate and hence $C$ is contained in the normal subgroup $H$. Moreover since $H / H \cap K \cong K C / K \cong C, H \cap K$ is complemented in $H$ by $C$.

LEMMA 3.2.2. Let $W=A$ wr $B$ with $A$ and $B$ finite groups of coprime order. Then any normal nilpotent subgroup of $W$ is contained in $K$.

Proof. We show that a normal subgroup $H$ which is not contained in $K$ cannot be nilpotent by producing two elements of coprime order which fail to commute. 
Let $C \neq 1$ be a complement for $H \cap K$ in $H, C$ a subgroup of $B$. Note that $H \cap K$ $\neq 1$, else the normal subgroup $H$ would be contained in $B$ which is impossible. Let $c$ be a nontrivial element of $C$ and let $g \in K$ be defined by choosing $g(c)$ to be a nontrivial element $a$ of $A$ and $g(b)=1$ for all $b \neq c$ in $B$. The commutator $h=[g, c]$ $\in H \cap K$, hence has order prime to $c$; moreover $h(c)=g^{-1}(c) g^{c}(c)=a^{-1}$ while

$$
\begin{aligned}
h^{c}(c)=\left(g^{-1}\right)^{c}(c) g^{c^{2}}(c) & =1 & & \text { if } c^{2} \neq 1 \\
& =a & & \text { if } c^{2}=1
\end{aligned}
$$

Since $A$ and $B$ have coprime order we can insure that not both $a$ and $c$ have order two, hence $h \neq h^{c}$ and $H$ is not nilpotent.

Proof of theorem. Since $\Phi(K)=1$ if and only if $\Phi(A)=1$, one part of the proof is an immediate consequence of 2.6 .3 which states that $\Phi(K) \leqq \Phi(W)$. Conversely if $\Phi(A)=1$ then by 3.1.1 $\Psi(A)=S(A)_{\alpha}=Z_{p_{1}} \times \cdots \times Z_{p_{n}}$ for primes $p_{1}, \ldots, p_{n}$. Since $\Phi(K)=1$ by 3.1 .1 and 3.1.2 $K$ splits over $\Psi(K)=\Psi(A)^{(B)}$. Hence $\Psi(K)$ as a normal nilpotent subgroup of $W$ is contained in $\Psi(W)$. By Lemma 3.2.2 then $\Psi(W)$ $=\Psi(K)=\Psi(A)^{(B)}$ and since $W=K B=\Psi(W) K^{*} B$ where $\Psi(W) \cap K^{*}=1$ we have $\Psi(W) \cap K^{*} B=1$. In other words $W$ splits over $\Psi(W)$ so by virtue of 3.1.2 it is enough to show that $\Psi(W)=S(W)_{\alpha}$. Clearly $S(W)_{\alpha} \leqq \Psi(W)$. But $\Psi(W)=\Psi(A)^{(B)}$ $=\left(Z_{p_{1}} \times \cdots \times Z_{p_{n}}\right)^{(B)}=Z_{p_{1}}^{(B)} \times \cdots \times Z_{p_{n}}^{(B)}$, that is $\Psi(W)$ is a product of minimal normal subgroups of $W$ and is abelian, which implies the remaining inclusion and thus equality. This completes the proof of Theorem 3 .

3.3. Proof of Theorem 4. I am indebted to L. G. Kovacs for suggesting the following lemma.

LEMMA 3.3.1. Let $D$ be an abelian normal subgroup of a group $G$ and suppose $G$ splits over $D$. If $M$ is a maximal subgroup of $G$, either $M$ contains $D$ or $M \cap D$ is a maximal $G$ normal subgroup of $D$. Conversely, every maximal $G$ normal subgroup of $D$ occurs as $M \cap D$, where $M$ is a maximal subgroup of $G$.

Proof. Let $C$ be a complement for $D$ in $G$ and let $M$ be maximal in $G$. If $D \leq M$ then $M D=G$; hence $M \cap D \triangleleft G$. If $M \cap D<N \leqq D$ with $N \triangleleft G$ then $M<N C$. For if $m=d c(d \in D, c \in C)$ is an arbitrary element of $M$, then since $N \$ M$ we have $N M=G$; hence $d=n m_{1}\left(n \in N, m_{1} \in M\right)$ and thus $m_{1}=n^{-1} d \in M \cap D<N$ from which it follows that $d \in N$ and $m \in N C$. That $M<N C$ follows from the fact that $M \cap D<N$. We conclude that $N C=G$ and $N=D$.

Now suppose $H$ is a maximal $G$ normal subgroup of $D$. If $d \in D \backslash H$, then $n m_{G}(d, H)=D$ which implies, since $D$ is abelian, that $\langle d, H C\rangle=G$, from which it follows that $H C$ is maximal in $G$.

For convenience we restate

TheOREM 4. Let $A$ and $B$ be groups without maximal subgroups, with $A$ abelian. Then $W=A \operatorname{Wr} B(A$ wr $B)$ has no maximal subgroups. 
Proof. We assume $W$ contains a maximal subgroup $M$ and look for a contradiction. If the base group $K$ is contained in $M$ then $M=K(M \cap B)$; hence there exists a proper subgroup $B_{1}$ of $B$ such that $M \cap B<B_{1}$ and $M<K B_{1}$. So $K \$ M$ and by Lemma 3.3.1 $K \cap M$ is a maximal $W$ normal subgroup of $K$, which implies that $\pi_{b}(K \cap M)=\pi_{b^{\prime}}(K \cap M)$ for all $b, b^{\prime} \in B$. Let $\pi_{b}(K \cap M)<L<A$. Then $K \cap M<L^{(B)} \triangleleft W$, a contradiction which establishes the theorem.

\section{REFERENCES}

1. W. Gaschütz, Uber die $\Phi$-Untergruppe endlicher Gruppen, Math. Z. 58 (1953), 160-170.

2. G. Higman and B. H. Neumann, On two questions of Itô, J. London Math. Soc. 29 (1954), 84-88.

3. W. Magnus, A. Karrass and D. Solitar, Combinatorial group theory, Interscience, New York, 1966.

4. D. H. McLain, Finiteness conditions in locally soluble groups, J. London Math. Soc. 34 (1959), 101-107.

5. B. H. Neumann, An essay on free products of groups with amalgamations, Philos. Trans. Roy. Soc. London Ser. A 246 (1954), 503-554.

City University of New York, Hunter College

New York, New YorK 